\title{
Implicaciones y Efectos Neurológicos en el Desarrollo del Lenguaje
}

\section{Neurological Implications and Effects on Language Development}

Viriam Mejías-Padilla ${ }^{1}$

\section{Edición Especial: ENSAYOS}

Recibido: 20 de octubre de 2020

Aceptado: 24 de enero de 2021

Publicado: 28 de marzo de 2021

Institución:

IIII

${ }^{1}$ Universidad Hispanoamericana

Costa Rica

\section{E-mail / ORCID:}

$\Sigma_{0}$

vmejias@ccss.sa.cr

(D) https://orcid.org/0000-0001-8778-5450

\section{Citar así:}

\section{CSAPA / IEEE}

Mejías-Padilla, V. (2021). Implicaciones y Efectos Neurológicos en el Desarrollo del Lenguaje. Revista Tecnológica-Educativa Docentes 2.0, 8(1), 25-31, https://doi.org/10.37843/rted.v10i1.17 8

V. Mejías-Padilla. "Implicaciones y Efectos Neurológicos en el Desarrollo del Lenguaje". RTED, 10, No. 1, pp. 25-31, Abr. 2021

\section{Resumen}

El lenguaje, desde su concepción como sistema de comunicación se muestra implicado en todos los ámbitos del ser humano. En particular, en los procesos educativos, donde constituye la base de cualquier aprendizaje. Pese a esto, se ha investigado poco sobre los trastornos del lenguaje a nivel neurológico. Con este objetivo, el presente artículo llevó adelante un estudio teórico respecto de los caracteres neuronales implicados en el desarrollo del habla humano, así como los problemas más comunes que se presentan en este ámbito. De esta forma se buscó brindar a los educadores un sustento teórico que les permita orientar sus procedimientos a la hora de tratar condiciones de este tipo en los estudiantes de la educación formal, sobre todo mediante estrategias didácticas basadas en la plasticidad del cerebro y su capacidad para ser moldeado.

Palabras clave: Implicaciones, efectos, neurológicos, desarrollo, lenguaje.
Keywords: Disorders, language, neuronal, neuroplasticity, stimulation, education.

\begin{abstract}
From its conception as a communication system, language is shown to be involved in all areas of the human being. In particular, in the educational processes, where it forms the basis of any learning. Despite this, little research has been done on language disorders at the neurological level. With this objective in mind, this article carried out a theoretical study regarding the neural characters involved in the development of human speech and the most common problems that arise in this area. In this way, it was sought to provide educators with theoretical support that allows them to guide their procedures when treating conditions of this type in students of formal education, especially through didactic strategies based on the plasticity of the brain and its ability to be molded.
\end{abstract}




\section{Implicaciones y Efectos Neurológicos en el Desarrollo del Lenguaje}

\section{Introducción}

El lenguaje constituye la base fundamental de los procesos de socialización en el ser humano, al mismo tiempo que un aspecto primordial en la educación formal. En ese sentido, el desarrollo del habla se considera indispensable en el proceso de aprendizaje del individuo, siendo uno de los pilares para su interacción e inclusión en los ámbitos académico, laboral, familiar o social (Agudelo, 2007). Un inadecuado desarrollo del lenguaje puede afectar de manera importante aspectos significativos en la vida del individuo, incluyendo su desempeño educativo.

En la educación formal se cuenta con estrategias $o$ estimulaciones que buscan corregir aquellas problemáticas a nivel de léxico que se derivan de condiciones externas al menor. Sin embargo, es poca la información $\mathrm{y}$ herramientas con que cuentan los docentes en relación con los problemas de lenguaje que derivan de condiciones internas, tal como es el caso de aquellas cuyas causas son neuronales.

El presente artículo busca un acercamiento teórico a las implicaciones del desarrollo neurológico en el proceso de formación del lenguaje en infantes. Esto tomando en cuenta las etapas de construcción de habilidades relacionadas con el aspecto motriz o cognitivo, las que condicionan todo el proceso de desarrollo del lenguaje, afectando los procesos educativos y sociales a los cuales se enfrentarán los individuos durante su edad adulta. (Millá \& Mulas, 2004).

El interés por abordar estas implicaciones en las edades tempranas del individuo parte de considerar esta etapa como aquella en que se da un mayor desarrollo neuronal y del lenguaje. Se trata de un período en el cual el cerebro posee una mayor capacidad para aprender debido a que cuenta con una mayor cantidad de conexiones entre neuronas.

Así, el presente ensayo tuvo como objetivo estudiar las implicaciones o efectos neurológicos presentes en el desarrollo del lenguaje, a fin de conocer cómo estas afectan el proceso educativo u otras áreas del desarrollo humano. De esta manera se pretende unamejor comprensión del proceso de estructuración del habla desde las edades tempranas, tanto como las áreas del cerebro involucradas y las estrategias de aprendizaje más apropiadas a la hora de tratar los trastornos neurolingüísticos.

\section{Desarrollo}

\section{Importancia del lenguaje en la educación}

Para Maturana (2020, p.7) el lenguaje no sólo refiere a su concepción tradicional, como sistema simbólico de comunicación fundado en su mayoría sobre sonidos o palabras, sino también a la unión de estas últimas con un conjunto mucho mayor de símbolos, en el cual los gestos son una parte activa dentro del proceso de comunicación. Agrega este autor que este tipo de interacciones dentro del habla se da por intermedio de coordinaciones de acción consensuales.

En cuanto al adecuado desarrollo del lenguaje dentro del proceso educativo es importante hacer notar que dicho proceso resulta esencial para el ser humano, al ser una base de representación, comunicación $\mathrm{y}$ socialización (González, 2003, p.16). De ahí que se considera al lenguaje como una actividad compleja, en la que se implican una serie de elementos por los cuales el individuo es capaz de comunicarse con otros semejantes. De ahí que Niño (1994) refiera al lenguaje como aquella capacidad de abstracción de la realidad cuyo objetivo es comunicarse por cualquier medio.

En el ámbito académico, el lenguaje es fundamental para que los procesos de lectoescritura se desenvuelvan de una forma adecuada. De ahí que Fernández (2015) mencione el lenguaje como un proceso diverso, el cual es indispensable evaluar en edades tempranas, a fin de detectar problemas que puedan afectar el desarrollo normal del infante en su proceso educativo.

De ahí surge la necesidad de comprender los factores neurológicos inmersos en el proceso de adquisición del lenguaje, a fin de detectar, abordar y corregir de forma temprana posibles irregularidades. 


\section{Implicaciones y Efectos Neurológicos en el Desarrollo del Lenguaje}

Por otra parte, resulta importante el aporte de Gallardo (2016), quien recalca la importancia de la adquisición del lenguaje, su estimulación o motivación, para el desarrollo efectivo en los niños. Diferentes pedagogías tales como el constructivismo, la pedagogía conceptual y la pedagogía crítica ubican al lenguaje como una base primordial en la educación.

Así mismo, Martínez (2004) sostiene que el proceso educativo resulta básico en el desarrollo del lenguaje, en tanto proceso mediante el cual el estudiantado desarrolla una serie de competencias sobre la comprensión y el aprendizaje, entre otros.

Por tanto, es fundamental el adecuado desarrollo del lenguaje durante los primeros años de vida del individuo, como herramienta fundamental para su adecuada incorporación al proceso educativo formal, y por ende, para su éxito académico.

\section{Desarrollo del lenguaje}

Como se mencionó antes, el "desarrollo del lenguaje es un proceso complejo en el cual intervienen diferentes factores" (García \& Calatrava, 2008, p. 1), tanto internos como externos.

Sobre los factores internos, lo que son competencia de esta investigación, cabe resaltar que durante los primeros años de vida del ser humano el cerebro se encuentra en una etapa de plasticidad gracias a la cual el sistema nervioso desarrolla aquellas áreas encargadas de desempeñar las diferentes funciones necesarias para sobrevivir. Además, es antes de los dos años de edad cuando se forman las ramificaciones neuronales encargadas del proceso de comunicación, así como para la recepción de mensajes, lo que resulta indispensable en el desarrollo del lenguaje a la vez que incide en los procesos de aprendizaje formal. (Rodríguez, 2014).

En ese sentido, existen procesos innatos dentro del desarrollo del habla, que derivan de una estructura anatómica-funcional destinada para este propósito. Y sin embargo, no es posible desarrollar un sistema comunicativo sin un adecuado desarrollo neuronal que lo impulse (Mauri \& Privadilla, 2013).

Para Shaffer \& Kipp (2007), desde una teoría interaccionista se fundamenta una preparación biológica previa al desarrollo del lenguaje, cuyo proceso se ve reflejado en un sistema nervioso que madura progresivamente. En esta etapa de maduración el ambiente posee gran relevancia, ya que es gracias a este que inicia la adquisición de diferentes habilidades relacionadas con el lenguaje. Esta teoría justifica la conexión del área de Broca (encargada del ordenamiento de fonemas en palabras y oraciones) con el área de Wernicke (cuya función consiste en procesar los sonidos del habla para una adecuada comprensión del lenguaje), tanto como muestra una estrecha relación entre el adecuado desarrollo del área de Wernicke, primera en desarrollarse con el proceso de escucha, y el área de Broca, en la cual se determinará una adecuada adquisición de lectoescritura. De existir carencias en la estimulación externa podrían presentarse deficiencias a nivel de léxico, ya que es por medio del contexto donde el menor conoce o procesa el significado de cada palabra.

\section{Trastornos del lenguaje desde la neuroplasticidad}

La neuroplasticidad es la capacidad del cerebro para modelar su estructura en consideración a las experiencias del individuo, a su contexto, así como por los procesos de aprendizaje a que se encuentra expuesto. Esto gracias al proceso de sinaptogénesis (nacimiento de nuevas neuronas) así como a podas sinápticas (muerte de neuronas no útiles).

Esta plasticidad cerebral está presente desde el nacimiento de individuo, extendiéndose durante todo el desarrollo y disminuyendo progresivamente a través de los años. Es por esta razón que resulta importante intervenir los trastornos del lenguaje desde edades tempranas, a fin de aprovechar que los sistemas neuronales son más susceptibles a los estímulos y que es posible generar cambios o desarrollar habilidades gracias a esa 


\section{Implicaciones y Efectos Neurológicos en el Desarrollo del Lenguaje}

estimulación (Howard- Jones, 2011).

Gracias a esta capacidad de regeneración cerebral resulta posible atender las irregularidades neurolingüísticas por intermedio de la estimulación, modificando las estructuras mentales para beneficiar el desarrollo e incremento de habilidades relacionadas con la comunicación. De ahí que Aguilar (2002) mencione que "la memoria y el aprendizaje surgen del estímulo realizado dentro de los procesos plásticos del cerebro, en donde se modifican las vías neuronales que se comunican con otras" (p. 55).

De este contexto deriva la necesidad de que a nivel educativo, sobre todo durante el primer ciclo de educación primaria, se implemente una adecuada estimulación del lenguaje mediante estrategias de aprendizaje que resulten coherentes con este objetivo.

\section{Trastornos neuronales que afectan el correcto desarrollo del lenguaje}

A nivel educativo es necesario que el docente sea capaz de identificar si una afectación del habla deriva de factores internos o externos, a fin de establecer adecuadamente algunas estrategias para tratar tal condición.

En ese sentido cabe mencionar que los trastornos por factores externos más comunes se dan en el área de semántica o léxico del infante, siendo que por lo común tienen que ver con el significado individual de cada palabra, el cual surge por la interacción con otros hablantes dentro del contexto, por lo general con los miembros de familia.

Por ello, estos trastornos son derivados de una poca o nula estimulación del infante, en especial desde los 3 a los 5 años de edad, periodo fundamental para concluir el desarrollo del léxico y gracias a que el individuo logra internalizar el significado de nuevos conceptos.

Por otra parte, los trastornos receptivoexpresivos, por factores internos, se caracterizan por una alteración tanto del lenguaje receptivo como del expresivo. Por lo general derivados de déficits en distintas áreas de procesamiento sensorial de información,

especialmente en el temporal auditivo (MINSAP, 1999). Estos déficits provocan dificultades en la comunicación, implicando tanto el lenguaje verbal como el gestual. (Fresneda \& Mendoza, 2005).

En cuanto a este tipo de alteraciones, Martínez (2008) menciona cinco trastornos principales: afasias, dispraxias, disartrias, disglosias, agnosias.

Sobre las afasias, este mismo autor (Martínez, 2008) refiere a once tipos diferentes, causados por una lesión o daño cerebral, en ocasiones congénito, que afecta la capacidad del individuo para producir o comprender el lenguaje $y$ por el cual se genera una fragmentación entre la palabra y el concepto.

Algunas afasias comunes en infantes son las siguientes:

1. Afasia global: se da por lesiones tanto en el área de Broca como de Wernicke, afectando todas las funciones del lenguaje. El menor no es capaz de producir ni comprender el significado o función del lenguaje, tanto a nivel escrito como oral.

2. Afasia de Broca o afasia motora: se manifiesta por un daño en el área de Broca, lo cual genera problemas a nivel articulatorio y de ahí un vocabulario restringido a frases cortas realizadas con gran esfuerzo. A nivel de comprensión también se dan fallas, aunque muy leves, lo cual puede generar disgrafías (trastornos en expresión escrita) y dislexias (trastornos en lectura).

3. Afasia de Wernicke o afasia sensorial: se origina por fallas en la zona de Wernicke, generando problemas de discriminación fonémica, de comprensión en sintáctica y semántica. Los niños con esta condición son capaces de articular frases y oraciones de forma fluida pero carentes de significado.

4. Anomia o afasia amnésica: este tipo de trastornos se caracteriza por una dificultad para encontrar palabras léxicamente apropiadas, por lo cual el 


\section{Implicaciones y Efectos Neurológicos en el Desarrollo del Lenguaje}

5. niño puede recurrir a utilizar palabras generales o expresiones deícticas sin afectación en la comprensión.

6. Afasia de conducción o síndrome de desconexión: se da igualmente por una afectación en el área de Broca y Wernicke, aunque sin una grave afectación en la comprensión. El déficit tiene que ver con la producción de lenguaje, siendo que el individuo es incapaz de reproducir lo escuchado.

7. Afasia mixta: se presentan en un mismo paciente tanto la afasia motora como la sensorial, con una diversidad de deficiencias propias tanto de una como de otra (p. 9-14).

Así mismo cabe señalar que la dispraxia, disartria o disglosia surgen por afecciones neurológicas en determinadas áreas cerebrales o canales relacionados con actividad motora. En ese sentido, estos trastornos se caracterizan por una afectación en la exteriorización del mensaje, no en su codificación. Esta forma de afasia se acompaña muy a menudo de dispraxia o disartria, lo cual genera condiciones más complejas, afectando en mayor medida el desarrollo del lenguaje. De ahí que una dispraxia afecta el control en la secuencia de sonidos, mientras que una apraxia afecta actividades motoras, comprometiendo los movimientos de los músculos de la cara, el sentido espacial, así como los movimientos de las manos.

Mientras tanto, la disartria refiere a aquellas afectaciones a nivel verbomotor, por lo cual el infante puede presentar alteraciones en la pronunciación de los diferentes sonidos, sin lograr controlar intensidad ni duración. Es una afección casi imperceptible y normalmente se asocia a una mala pronunciación de consonantes.

Finalmente, las agnosias se generan por trastornos en el reconocimiento de estímulos, pudiendo ser de tipo visual, auditiva o táctil. Entre estas se encuentran la sordera auditiva, en la cual el cerebro no es capaz de analizar los sonidos; la sordera verbal, por lo común del lado

izquierdo y que se relaciona directamente con el lenguaje, en especial con el reconocimiento de sonidos; la sordera por forma en palabras, la cual, como su nombre lo indica, no afecta la diferenciación entre fonemas aunque no permite reconocer las palabras; la sordera de palabra, en la cual se logra reconocer palabras pero no su significado; la agnosia fonológica, que afecta el léxico-fonológico, siendo que no se logra repetir aquellas palabras no conocidas.

\section{Mediación pedagógica}

En el plano educativo si bien el contexto o familia juega un papel fundamental en el tratamiento o detección de los trastornos del lenguaje, realmente muchos infantes arrastran problemas hasta la educación formal. De ahí que es fundamental la detección y tratamiento a tiempo de los déficits.

Aunque los trastornos del lenguaje pueden derivarse de diversas alteraciones o factores, como los mencionados, es imprescindible reconocer aquellas señales relacionadas con los trastornos neuronales del lenguaje (Moran et al, 2017).

Esta labor puede representar todo un reto para el docente, ya que intervienen aspectos no sólo neurológicos, sino también psicológicos y sociales. De ahí que se requiera una intervención global del problema, en la cual participen especialistas en cada ámbito, así como el núcleo familiar del menor.

A nivel educativo el diagnóstico inicial del estudiante con trastornos del lenguaje permitirá fijar alternativas metodológicas ajustadas a sus habilidades y condiciones: algunos de estos ajustes pueden inclusive consistir en actividades colaborativas o cooperativas, que propicien las interacciones sociales.

A la par de eso resulta necesario considerar los apoyos requeridos a nivel educativo, tales como la terapia de lenguaje, estableciendo una línea de trabajo conjunta con un especialista en esta materia.

De igual forma es importante considerar que las estrategias de estimulación pueden 


\section{Implicaciones y Efectos Neurológicos en el Desarrollo del Lenguaje}

mejorar algunas capacidades del menor, haciendo posible una intervención colectiva que estimule el lenguaje a nivel general.

En todos los casos se debe considerar la estimulación del lenguaje como aquellas acciones encaminadas, de forma consciente o justificada, a desarrollarlo, así como a potenciar las habilidades fonológicas, semánticas y pragmáticas (Fernández, 2011, p.5).

\section{Conclusiones}

Una adecuada formación del lenguaje durante los primeros años de vida resulta determinante para el futuro desempeño del individuo en el ámbito educativo. Por ello la necesidad, a nivel de educación primaria, de que se logre detectar este tipo de trastornos. De lo contrario se fuerza a los estudiantes con problemas neurológicos a cumplir con respuestas inadecuada a su condición, generando desinterés o bajo rendimiento.

Es importante recalcar que el buen rendimiento en el área del lenguaje constituye un indicador de éxito escolar, ya que estas habilidades facilitan el proceso de comunicación y a su vez las labores escolares.

Un docente que cuenta con conocimientos y herramientas básicas sobre los trastornos del lenguaje se encuentra mejor capacitado para lograr una detección temprana de irregularidades en el desarrollo neuronal de menor, pudiendo establecer estrategias de intervención acordes a cada caso.

El diagnóstico del desarrollo del lenguaje debe darse en los primeros años de educación primaria, aunque preferiblemente en la edad preescolar con una posterior evaluación en el primer ciclo. Mientras más pronta sea la detección del problema mayor probabilidad de revertir sus efectos mediante un adecuado proceso de intervención.

En este sentido se recomienda estimular el desarrollo de lenguaje gestual intencionado (por medio de estrategias) en los niños en edad escolar (I ciclo para efectos de este trabajo). Una correcta estimulación puede generar mejoras en el desempeño de los niños debido a la plasticidad cerebral que se tiene durante la infancia.

Pese a lo anterior, los beneficios de tipo de estimulación son patentes incluso si el proceso inicia cuando los niños ya han ingresado a primaria o llevan algunos años en ella (7- 10 años). Si bien a esta edad los menores ya poseen un nivel de desarrollo dependiente de sus experiencias y contexto, este no es definitivo, y mucho menos constituye una limitación para la mejora o el cambio respecto de sus habilidades para el lenguaje.

\section{Referencias}

Aguilar, F. (2002). Plasticidad Cerebral: Parte 1. Revista Medicina IMSS; 41 (1) pp. 55-64.

Agudelo, M.G. (2007). Importancia del lenguaje en el ámbito educativo. https://1library.co/document/download/wq2wjm2q\#_ $=$

Fernández-Pérez, M. (2015). Lenguaje infantil y medidas de desarrollo verbal. ENSAYOS, Revista de la Facultad de Educación de Albacete, 30(2).

Fernández, B. (2011). Estimulación cognitiva en niños de segundo ciclo de infantil. (Tesis de grado). https://repositorio.unican.es/xmlui/bitstream/handle/1 0902/1489/Fern\%C3\%A1ndez\%20Roiz,\%20Beatriz.p df?sequence $=1$

Fresneda, M. D., \& Mendoza, E. (2005). Trastorno específico del lenguaje: Concepto, clasificaciones y criterios de identificación. Revista de Neurología, 41(1), p. 51-56.

Gallardo Martínez, E. (2016). Inicio tardío del lenguaje: revisión general. Medigraphic: literatura biomédica, 3 (5), p. 89-96.

González, C. (2003). Enseñanza y aprendizaje de la lengua en la escuela infantil. Granada: Grupo Editorial Universitario, D.L.

García, A. y Calatrava, M. (2008). Bases anatómicas, fisiológicas y neurológicas del lenguaje. su importancia en la intervención en el aula de audición y lenguaje. Innovación y experiencias (11). https://archivos.csif.es/archivos/andalucia/ensenanza/r evistas/csicsif/revista/pdf/Numero_11/VARIOS_BAS ES.pdf

Howard-Jones, P. (2011). ¿Qué tiene que ver la neurociencia con la educación? En P. Howard-Jones, Investigación Neuroeducativa. Neurociencia, Educación y cerebro: De los contextos a la práctica (26-50). La Muralla, S.A. 


\section{Implicaciones y Efectos Neurológicos en el Desarrollo del Lenguaje}

Martínez, M (2004) El procesamiento multinivel del texto escrito ¿un giro discursivo en los estudios sobre la comprensión de textos? Lenguaje (32), Escuela de ciencias del Lenguaje, Universidad del Valle.

Martínez, J.M. (2008) Neurolingüística: patologías y trastornos del lenguaje. Revista Digital Universitaria. 9 (12), pp. 9-14.

http://www.revista.unam.mx/vol.9/num12/art103/art1 03.pdf

Maturana, H. (2020) Emociones y Lenguaje en Educación y Política. Centro de Educación del Desarrollo (CEO). 5ta. Edición. Ediciones Pedagógicas Chilenas S. A.

Millá, M.G. \& Mulas, F. (2014) Atención temprana y programas de intervención específica en el trastorno del espectro autista. Revista de neurología. 48, (2), pp. $47-52$

Morán, M. R., Vera, L. Y., \& Morán, M. R. (2017). Los trastornos del lenguaje y las Necesidades Educativas Especiales. Consideraciones para la atención en la escuela. Universidad y Sociedad, 9(2), 191-197. Recuperado de http://rus.ucf.edu.cu/index.php/rus

Mauri, S. \& Rivadulla, J. (2013). Bases fisiológicas del proceso de adquisición del lenguaje. Aprendizaje y plasticidad. (Tesis de grado). Recuperado de: https://ruc.udc.es/dspace/bitstream/handle/2183/11854 /MauriMartinez_Sara_TFG_2013.pdf?sequence $=2$ \&is Allowed $=\mathrm{y}$

MINSAP. (1999). Manual de prácticas clínicas para la atención integral a la salud de la adolescencia. Ministerio de Salud Pública de Cuba.

Niño, M. (1994). Los procesos de la comunicación y del lenguaje. Ecoe Ediciones

Ramírez, C. (2014). La influencia de la familia y la escuela en el desarrollo del lenguaje oral en niños de 3 a 5 años a través de la aplicación de un programa de estimulación del lenguaje (Doctoral disertación). Universidad Autónoma de Madrid.

Rodríguez, A. (2014). La inteligencia en educación infantil articulada por la herencia, ambiente escolar, social y familiar. Editorial: Liber Factory.

Samper, M (2002) ¿Qué es pedagogía conceptual? Educación y Cultura, (59), FECODE.

Shaffer, D \& Kipp, K. (2007). Desarrollo del lenguaje y de las habilidades comunicativas. En D, Shaffer \& K, Kipp, Psicología del Desarrollo: Infancia y adolescencia. (379-418). Thomson Editores, S.A. 